\title{
Acknowledgement for Reviewers
}

\author{
Bibliography \\ DOI http://dx.doi.org/10.1055/s-0044-100484 \\ Int J Sports Med 2018; 39: 154-157 \\ (c) Georg Thieme Verlag KG Stuttgart · New York \\ ISSN 0172-4622
}

\begin{abstract}
Good reviewers are essential to the success of any journal and peer review is a major pillar of science. We are grateful to those mentioned below to have dedicated their time and expertise to help our authors improve and refi ne their manuscripts and support the Editor(s) in the decision making process in the past year.
\end{abstract}

The following received out Top Reviewer of the Year award:

Pascal Edouard, France

Antonio Soares, Brazil

Cristine Alberton, Brazil

We wish all our readers, reviewers, authors and friends a happy and prosperous new year!

José Alberto Ramos Duarte

Editor-in-Chief

International Journal of Sports Medicine

Georg Thieme Verlag Publisher

Abdel-aziem Amr Almaz, Egypt

Abe Takashi, United States

Aboshkair Kamil, Malaysia

Abrahams Shameemah, South Africa

Adamczyk Waclaw, Poland

Ahmetov Ildus, Russian Federation

Akagi Ryota, Japan

Akam Elizabeth, United Kingdom

Akazawa Nobuhiko, Japan

Akca Firat, Turkey

Akima Hiroshi, Japan

Akimoto Takayuki, Japan

Alberton Cristine, Brazil

Aldred Sarah, United Kingdom

Alemdaroglu B. Utku, Turkey

Almeida Leonardo, Brazil

Almeida Matheus, Brazil

Alp Ebru, Turkey

Ammar Achraf, Tunisia

Andrade Ricardo J., France

Andreato Leonardo, Brazil

Angius Luca, United Kingdom

Annino Giuseppe, Italy

Appell Coriolano Hans-Joachim, Germany
Arampatzis Adamantios, Germany

Araújo Joamira, Brazil

Arcuri Juliano, Brazil

Areta Jose, Norway

Arnold Rachel, United Kingdom

Arratibel-Imaz Iñaki, Spain

Artioli Guilherme, Brazil

Aslankeser Zübeyde, Turkey

Astorino Todd, United States

Aucouturier Julien, France

Augustine Jacqueline, United States

Ayala Francisco, Spain

Azevedo Paulo Henrique Silva, Brazil

Babault Nicolas, France

Bacon Catherine, New Zealand

Baker Robert L., United States

Balci Sükrü, Turkey

Balshaw Tom, United Kingdom

Barberio Matthew, United States

Barnes Jeremy, United States

Barnes Richard, United Kingdom

Barrero Anna, Spain

Barrett Stephen, United Kingdom

Barroso Renato, Brazil
Bartolomei Sandro, Italy

Barwood Martin, United Kingdom

Basset Fabien, Canada

Baudry Stephane, Belgium

Bazzucchi Ilenia, Italy

Behrens Martin, Germany

Belavý Daniel, Germany

Bellar David, United States

Bellenger Clint, Australia

Belski Regina, Australia

Benton Melissa, United States

Bergstrom Haley, United States

Berli Corina, Switzerland

Bermon Stéphane, Monaco

Berzosa Cesar, Spain

Betsch Marcel, United States

Bezodis Neil, United Kingdom

Bigley Austin, United States

Billaut Francois, Canada

Björklund Glenn, Sweden

Black Christopher, United States

Blasco-Lafarga Cristina, Spain

Bloch Wilhelm, Germany

Bogdanis Gregory, Greece 
Boguszewski Dariusz, Poland

Bonello Spiteri Danica, Malta

Bonganha Valéria, Brazil

Boolani Ali, United States

Borges Fernando, Brazil

Borges Juliana, Brazil

Boris Jidovtseff, Belgium

Bosch Tyler, United States

Bossi Arthur Henrique, United Kingdom

Bottaro Martim, Brazil

Boudreau Shellie, Denmark

Bowen Scott, Germany

Bragada Jose, Portugal

Braun William, United States

Bray James, United Kingdom

Bresciani Guilherme, United States

Briggs Matthew, United States

Brooks George A., United States

Brown Cathleen, United States

Brown Lee, United States

Bueno Jr Carlos, Brazil

Burger Marilize, South Africa

Bye Elizabeth, Australia

Caderby Teddy, Réunion

Cadore Eduardo, Brazil

Cambridge Edward, Canada

Capranica Laura, Italy

Carregaro Rodrigo, Brazil

Caruso Flavia, Brazil

Castagna Olivier, France

Castellano Julen, Spain

Castro Flávio, Brazil

Cazzola Dario, United Kingdom

Celes Rodrigo, Brazil

Chang Hsiao-Yun, Taiwan

Chang Yu-Jen, United States

Chen Lin, China

Chrzanowski-Smith Oliver, United Kingdom

Chtourou Hamdi, Tunisia

Chughtai Morad, United States

Ciccone Anthony, United States

Cigrovski Vjekoslav, United States

Ciria Luis F., Spain

Coelho Eliza, Brazil

Cole Matthew, United Kingdom

Coleman Damian, United Kingdom

Collins Malcolm, South Africa

Cometti Carole, France

Connes Philip, Guadeloupe

Coratella Giuseppe, Italy

Costa Vitor, Brazil

Cotton Clark, United States

Cramer Matthew, United States

Crisafulli Antonio, Italy

Cross Kevin M., United States

Curty Victor, Brazil

Cyrino Edilson, Brazil da Silva Cristiano, Brazil

da Silva Edson, Brazil

Dai Boyi, United States

Dalecki Marc, United States

Damas Felipe, Brazil

Daneshjoo Abdolhamid, Malaysia

Daussin Frédéric, France

de Ruiter Cornelis J., United States

de Ste Croix Mark, United Kingdom

de Vries Wouter, Netherlands

de Witt John K, United States

Della Porta Giovanna, Portugal

Deminice Rafael, Brazil

Denham Joshua, Australia

Denis Romain, France

DeWitt Terry, United States

Dhahbi Wissem, Tunisia

Di Michele Rocco, Italy

Dias Raphael, Brazil

Diaz German, Spain

Díaz Víctor, Switzerland

Diel Patrick, Germany

Diffendaffer Alek, United States

Dixon Warren E., United States

Doering Thomas M., Australia

Donovan Luke, United States

Dornelas de Andrade Armele, Brazil

Dotan Raffy, Canada

Driss Tarak, France

Drozdovska Svitlana, Ukraine

Drust Barry, United Kingdom

Duarte José, Portugal

Duc Sébastien, France

Earnest Conrad, United States

Edouard Pascal, France

Eleni Dimakopoulou, Greece

Elliott Adrian, Australia

Esposito Fabio, Italy

Eston Roger, Australia

Evanoff Nicholas, United States

Exell Tim, United Kingdom

Eynon Nir, Australia

Faiss Raphael, Switzerland

Farinatti Paulo, Brazil

Faulhaber Martin, Austria

Ferley Derek D., United States

Fernandes Ricardo, Portugal

Fernandez-Gonzalo Rodrigo, Sweden

Ferreira Rita, Portugal

Ferreira-Junior João Batista, Brazil

Filho Dario, Brazil

Filliau Christophe, France

Finestone Aharon S., Israel

Floria Pablo, Spain

Fonseca Helder, Portugal

Formenti Damiano, Italy

Fortington Lauren, Australia
Foster Josh, United Kingdom

Fradkin Andrea, United States

Franchini Emerson, Brazil

Franzoni Ferdinando, Italy

Fruer-Zakrzewski Julia, United Kingdom

Fukuda David, United States

Galbraith Andy, United Kingdom

Galloway Stuart, United Kingdom

García-García Oscar, Spain

Gatterer Hannes, Austria

Gautrey Charlotte, United Kingdom

Geijer Justin, United States

Gentil Paulo, Brazil

Gerosa-Neto José, Brazil

Gil Susana, Spain

Girgis Beshoy, Portugal

Gomes-Santos Igor, Brazil

Gonçalves Daniel, Portugal

Gonzales Joaquin, United States

Goosey-Tolfrey Victoria, United Kingdom

Goto Kazushige, Japan

Goulet Eric, Canada

Gribble Phillip, United States

Gulliver Amelia, Australia

Gündüz Filiz, Turkey

Guppy Fergus, United Kingdom

Gutierrez Gregory, United States

Haag Samuel, United States

Haakonssen Eric, Australia

Hagberg James, United States

Hagovská Magdaléna, Slovakia

Haile Luke, United States

Hamill Joe, United States

Hamlin Michael, New Zealand

Hanson Nicholas, United States

Haraldsdottir Kristin, United States

Hartmann Ulrich, Germany

Haugen Thomas, Norway

Havenith Georges, United Kingdom

Hawkins Steven, United States

Hayes Phil, United Kingdom

Hazell Tom, Canada

Hedman Kristofer, Sweden

Heikenfeld Roderich, United States

Herda Trent, United States

Hermassi Souhail, Tunisia

Hernando Carlos, Spain

Hiley Mike, United Kingdom

Hiraoka Koichi, United States

Hirata Rogerio, Denmark

Hizume-Kunzler Deborah, Brazil

Hofmann Peter, Austria

Hollander Karsten, Germany

Holmberg Hans-Christer, Sweden

Holmstrup Michael, United States

Hoppe Matthias, Germany

Horsak Brian, Austria 
Howe Louis, United Kingdom Howell David, United States Hunter Gary, United Kingdom Hurst Christopher, United Kingdom Jacquemet Vincent, Canada Jakeman John, United Kingdom Jakovljevic Vladimir, Serbia Jaric Slobodan, United States Jarvinen Tero, Finland Jelkmann Dr. med. W., Germany Jensen Randall, United States Jobson Simon, United Kingdom Junior Nevton, Brazil Kanaley J.A., United States Kanegusuku Hélcio, Brazil Karsten Bettina, United Kingdom Kato Morimasa, Japan Kavazis Andreas, United States Kawata Keisuke, United States Kellis Eleftherios, Greece Kennedy Rodney, United Kingdom Khlopas Anton, United States Kiefer Adam, United States Kiely John, United Kingdom Kinugasa Ryuta, United States Kluser Allan, Brazil Knechtle Beat, Switzerland Knobe Matthias, Germany Koh Yunsuk, United States Köklü Yusuf, Turkey Konishi YU, Japan Konstantinidou Anna, Greece Kotzamanidis Christos, Greece Koutlianos Nikolaos, Greece Kovács Attila, Hungary Kraft Justin, United States Krueger Karsten, Germany Krysztofiak Hubert, Poland Kuepper Thomas, Germany Kujawski Slawomir, Poland Kuo Chia-Hua, Taiwan Kusy Krzysztof, Poland Lacourpaille Lilian, France Layne Andrew, United States Layton Aimee, United States Lazzer Stefano, Italy Leddy John, United States Lee C. Matthew, United States Lee Chia-Lun, Taiwan Legaz-Arrese Alejandro, Spain Leicht Anthony, Australia Leite Tailce, Brazil Lepers Romuald, France Leppänen Mari, Finland Liepa Agris, United Kingdom Lin Wan-Teng, Taiwan Lira Fábio, Brazil
Little Jonathan, Canada

Loenneke Jeremy, United States

López Miñarro Pedro Ángel, Spain

Lopez-Villegas Antonio, Norway

Loturco Irineu, Brazil

Loubert Peter V., United States

Lucia Alejandro, Spain

Luz Coelho Rogerio, Brazil

Macaluso Andrea, Italy

Machado Fabiana, Brazil

MacInnis Martin, Canada

Maeo Sumiaki, Japan

Maffulli Nicola, United Kingdom

Magalhaes Flavio de Castro, United States

Malin Steve, United States

Marocolo Moacir, Brazil

Martel Gregory, United States

Martin-Hernandez J., Spain

Matsakas Antonios, United Kingdom

Mayo Xián, Spain

McCurdy Kevin, United States

McDaniel John, United States

McGuigan Polly, United Kingdom

McNaughton Lars, United Kingdom

Medeiros Hugo, Brazil

Mendes Romeu, Portugal

Meng Meimei, China

Mercer John, United States

Metcalfe Richard, United Kingdom

Michal Lehnert, Czech Republic

Mikulic Pavle, Croatia

Mills Chris, United Kingdom

Minahan Clare, Australia

Miyamoto Gisela, Brazil

Mizuno Takamasa, Japan

Mooses M., Estonia

Moraes Milton Rocha, Brazil

Moran Jason, United Kingdom

Mora-Rodriguez Ricardo, Spain

Moreira Alexandre, Brazil

Mota Maria, Portugal

Munguia-Izquierdo Diego, Spain

Muyor José, Spain

Naclerio Fernando, United Kingdom

Nakamura Fábio, Brazil

Nakamura Masatoshi, Japan

Natsis Konstatinos, Greece

Neto Gabriel, Brazil

Neville Jono, New Zealand

Nie Jinlei, China

Nikolaidis Pantelis, Greece

Nordsborg Nikolai, Denmark

Nuñez Javier, Spain

Ogasawara Riki, Japan

Okamoto Takanobu, Japan

Okita Koichi, United States

Oliva Francesco, Italy
Oliveira-Silva Iransé, Brazil

Ono Takashi, Japan

Oosthuyse Tanja, South Africa

Ortiz Alexis, United States

Ostrem Joseph, United States

Pareja-Galeano Helios, Spain

Parnell jill, United States

Paschalis Vassilis, Greece

Patikas Dimitrios, Greece

Peck Evan, United States

Peng Hsien-Te, Taiwan

Pereira Rogério, Portugal

Perkin Oliver, United Kingdom

Persson Andreas, United States

Petridou Anatoli, Greece

Pettitt Robert, United States

Philippon Marc, United States

Piacentini Maria Francesca, Italy

Pimenta Eduardo, Brazil

Pope Zachary, United States

Pourkazemi Fereshteh, Australia

Prieske Olaf, Germany

Prill Robert, Germany

Prior David, Australia

Qin Ling, Hong Kong

Rama Luis, Portugal

Ramos Joyce, Australia

Ratamess Nicholas, United States

Ratel Sebastien, France

Reeser Jonathan, United States

Reinert Andrew, United States

Remígio Bruno, Brazil

Rey Ezequiel, Spain

Riera Florence, France

Ritter Ophélie, France

Roberts Andrew J., United Kingdom

Roberts Simon, United Kingdom

Robertson Dr. Robert J., United States

Robinson Mark, United Kingdom

Rocha-Vieira Etel, Brazil

Rodríguez-Marroyo Jose, Spain

Rodríguez-Rosell David, Spain

Roelands Bart, Belgium

Roper Jenevieve, United States

Rothschild Carey, United States

Rousanoglou Elissavet, Greece

Rowe James, United States

Ruhe Alexander, Australia

Ryan Terence, United States

Ryder Justin, United States

Saglam Melda, Turkey

Saito Akira, Japan

Saji Naoki, Japan

Sanchis-Moysi Joaquin, Spain

Sandbakk Øyvind, Norway

Sandel Natalie, United States

Sanders Dajo, United Kingdom 
Santana Hugo A. P., Brazil

Santos Júlia, United States

Santos-Concejero Jordan, South Africa

Sardinha Luis, Portugal

Scheer Volker, Germany

Scheuermann Barry, United States

Schiavi Paolo, Italy

Scibora Leslie, United States

Scully David, United Kingdom

Sfyri Peggy, United Kingdom

Shield Anthony, Australia

Shimojo Guilherme, Brazil

Silva João Renato, Portugal

Simpson Andrew, United Kingdom

Singh Vincent, United Kingdom

Skinner lan, Australia

Smilios Ilias, Greece

Soares Antonio, Brazil

Sole Gisele, New Zealand

Souissi Nizar, Tunisia

Sousa Nelson, Portugal

Sousa Nuno, Brazil

Souza Raphael, Brazil

Stafilidis Savvas, Austria

Stanula Arkadiusz, Poland

Stebbins Charles, United States

Steinacker Jürgen, Germany

Stembridge Mike, United Kingdom

Stevens Tom Gerardus Antonia, Netherlands

Stickford Jonathan, United States

Stock Holly, United Kingdom

Stokes Keith, United Kingdom

Stoner Lee, New Zealand

Sugawara Jun, Japan
Sugisaki Norihide, Japan

Suijo Kenichi, Japan

Sullivan John S., United States

Supej Matej, Romania

Takai Yohei, Japan

Taniguchi K., United States

Temesi John, Canada

Tennent David, United States

Terada Masafumi, United States

Terrier Romain, France

Thomas Christopher, United Kingdom

Thompson Brennan, United States

Thompson Melissa, United States

Tian Ye, China

Till Kevin, United Kingdom

Timmins Ryan, Australia

Tomohiro YASUDA, Japan

Tompkins Marc, United States

Totosy de Zepetnek J. O., Canada

Toubekis Argyris, Greece

Trinity Joel, United States

Tsai Liang-Ching, United States

Turner Anthony, United Kingdom

Twist Craig, United Kingdom

Ueblacker Peter, Germany

Uhring J., United States

Uithoven Katelyn, United States

Vaara Jani, Finland

van den Tillaar Roland, Norway

van Guilder Gary, United States

van Meer Belle L., United States

Veeranki Sudhakar, United States

Vescovi Jason, Canada

Vicente-Salar Néstor, Spain
Vilas Boas Joao Paulo, Portugal

Vollaard Niels, United Kingdom

Wahl Patrick, Germany

Wakahara Taku, Japan

Wang Chun-Hou, Taiwan

Wang Jianxiong, Australia

Wang Ran, China

Watanabe Kohei, Japan

Wax Benjamin, United States

Westerterp K., Netherlands

Weston Kathryn, United Kingdom

Wild James, United Kingdom

Williams Sean, United Kingdom

Winder Brooke, United States

Wooten Joshua, United States

Wyatt Laura, United Kingdom

Yanci Javier, Spain

Ye Xin, United States

Yom Jae, United States

Zafeiridis Andreas, Greece

Zagatto Alessandro, Brazil

Zamunér Antonio Roberto, Brazil

Zaras Nikolaos, Greece

Zayni Richard, France

Zech Astrid, Germany

Zeller Sebastian, Germany

Zemková Erika, Slovakia

Zhanf Ying, China

Zhao Xing, United States

Zhu Qin, United States

Ziemann Ewa, Poland

Zimmer Philipp, Germany

Zotz Talita, Brazil 